\title{
Healthcare Costs for High-grade Glioma
}

\author{
YANG LIU ${ }^{1}$, EVAN TYLER ${ }^{2}$, MARTIN LUSTICK ${ }^{2}$, DAVID KLEIN ${ }^{3}$ and KEVIN A. WALTER ${ }^{1,4,5}$ \\ ${ }^{1}$ Department of Neurosurgery, ${ }^{4}$ Orthopedics, ${ }^{5}$ Wilmot Cancer Institute, School of Medicine and Dentistry, \\ ${ }^{3}$ Simon Graduate School of Business, University of Rochester, Rochester, NY, U.S.A.; \\ ${ }^{2}$ Excellus BCBS, Rochester, NY, U.S.A.
}

\begin{abstract}
Background/Aim: Data on verified healthcare costs for high-grade gliomas (HGGs) are limited. This study aimed to determine the healthcare costs for HGGs. Materials and Methods: A total of 88 primary HGGs patients diagnosed and treated at our Institution between 2011 and 2017 who had insurance plans administered with Excellus BCBS were retrospectively identified. Patient clinical information was linked with all verified insurance payment data. Results: Median insurance payments for clinical management of HGGs were $\$ 184,159.83$. The leading cost was therapeutic radiation oncology. Patients under commercial insurance had a longer survival time, and higher healthcare expenditures in total and in each phase of clinical care. Healthcare costs were higher during therapy initiation and at disease recurrence and lower during the interim. A generalized linear model showed that patients with commercial insurance, better Karnofsky Performance Status, and longer survival time had higher healthcare expenditures. Conclusion: Healthcare payments for HGGs patients were substantial and such high healthcare expenditures were positively associated with patient survival and commercial insurance.
\end{abstract}

Malignant gliomas account for $70 \%$ of malignant primary brain tumors diagnosed in the US every year and they continue to present a difficult therapeutic challenge (1-5). In addition to challenging clinical management, malignant brain tumor patients incur higher healthcare costs when compared both to healthy individuals, as well as patients with other

Correspondence to: Yang Liu, Department of Neurosurgery, School of Medicine and Dentistry, University of Rochester, Box 670, 601 Elmwood Ave, Rochester, NY, 14642, U.S.A. Tel: +15852758709 , Fax: +1 5852762892, e-mail: yang_liu@urmc.rochester.edu; Kevin A. Walter, Department of Neurosurgery, School of Medicine and Dentistry, University of Rochester, Box 670, 601 Elmwood Ave, Rochester, NY, 14642, U.S.A. Tel: +1 5852763581, Fax: +1 5852762892, e-mail: kevin_walter@urmc.rochester.edu

Key Words: High-grade gliomas, healthcare costs, insurance type, survival. forms of cancer (6-8). Total monthly costs for glioma patients can be 20 times more than for healthy controls ( 6 , 9). Previous studies to estimate the cost of glioma care have been based on commercial claims databases, however, such databases suffer several limitations including lack of detailed and verified clinical information $(6,10,11)$. Ray et al., estimated that healthcare costs for these patients were $\$ 106,896$ in the 6 months postindex surgery and $\$ 131,815$ in the 12 months postindex surgery (10). Similarly, Jiang et al. reported that total medical costs for 6 months and 12 months post GBM diagnosis were $\$ 160,974$ and $\$ 201,749$, respectively (11). Both of these studies suffer from the advantage and disadvantage of using a large commercial database. The large number of patients strengthens some estimates but it also prevents more granular examination and verification of individual patient data.

In addition, as US cancer care costs continue to increase (12-14), there is a movement toward bundled care and simplified payment schemes $(15,16)$. Data regarding the actual payments and costs associated with glioma care are critical to make informed decisions regarding healthcare management. Many previous studies of healthcare costs on high-grade gliomas (HGGs) were performed either before 2000 or used estimated data from Surveillance, Epidemiology and End Results (SEER) - Medicare or commercial claims database $(9-11,17,18)$. It is known that the treatment paradigm for patients with HGGs changed significantly since the introduction of Stupp regimen in 2005 (5) and data from SEER-Medicare or commercial claims database suffer the aforementioned limitations.

In this study, patients with primary HGGs diagnosed and treated at our Institution were retrospectively included and patient clinical information from electronic health record (EHR) was linked with relevant healthcare costs from the time of diagnosis to the time patients died or end of study from a single health insurance company, Excellus BlueCross BlueShield (BCBS) of Rochester, New York which managed both commercial and non-commercial (Medicare HMO, Medicaid HMO, etc.) products. We sought to investigate total healthcare costs for these patients, how these costs are 
distributed and any potential factors associated with these costs. The patient cohort involved 88 patients with over 44,000 separate insurance claims. This dataset represented a happy medium that is large enough to provide a good estimate of overall patient costs, but small enough to individually verify each patient diagnosis and treatment rather than relying on administratively coded national datasets.

\section{Materials and Methods}

Data source. This retrospective study was approved (protocol 00071072) by the University of Rochester Research Subjects Review Board with a waiver of informed consent. Adult patients (aged $\geq 18$ years) with initial diagnosis of HGGs (ICD-9: 191.XX and ICD-10: C71.XX) who were treated at our institution between January 1, 2011 and February 28, 2017 and who were under Excellus BCBS coverage during the entire study period were included. Clinical information for these patients were collected from EHR at our institution and matched to all payment data provided by Excellus BCBS. Overall survival time from the date of diagnosis to the date of death for any reason, or the date the patient was last known to be alive and relevant costs during the course of treatment were calculated. Healthcare costs were divided into three clinically relevant phases: 1), initiation care when surgery, radiation therapy and chemotherapy were implemented; 2), maintenance care when temozolomide or no chemotherapy was used and 3), recurrence care when new chemotherapy or radiation therapy was implemented.

Statistical analyses. Descriptive statistics were used to describe the demographic and clinical characteristics of the study cohort. Generalized linear model (GLM) with gamma distribution and log link function was conducted to examine potential factors associated with healthcare costs. Total healthcare costs were used as dependent variable and age, gender, Karnofsky Performance Status (KPS) score, married status, insurance type and survival as predictors. Wald chi-square was used for the model. All analyses were conducted using IBM SPSS Statistics (version 24).

Additionally, we ran two sensitivity analyses (SA) to ensure the results were not affected by analytical decisions. In SA1, tumor grade and the aforementioned predictors were included. In SA2, in addition to those predictors mentioned above, vital status was included.

\section{Results}

In the present study, 88 eligible patients with primary HGGs diagnosed and treated at our institution between January 1, 2011 and February 28, 2017 were identified and all claims data available for the study period were collected. Table I shows the demographic and clinical characteristics for these patients. Among them, $53.4 \%$ (47/88) were males while $46.6 \%(41 / 88)$ were females. The median age at diagnosis was 59 years (min. 30 years and max. 89 years). The majority of the patients were white $(94.3 \%, 83 / 88)$ with GBM $(80.7 \%, 71 / 88)$. Most of the patients $(72.7 \%, 64 / 88)$ had died (all causes) during the follow-up interval. The median survival time of these patients was 402 days (min. 37 days, max. 2059 days).
Table I. Demographic and clinical characteristics of the study cohort $(n=88)$.

\begin{tabular}{lc}
\hline Gender & \\
Male & $47(53.4 \%)$ \\
Female & $41(46.6 \%)$ \\
Age (year) & $59(30,89)$ \\
Median (min, max) & \\
Race & $83(94.3 \%)$ \\
White & $4(4.6 \%)$ \\
Non-white & $1(1.1 \%)$ \\
Unknown & $71(80.7 \%)$ \\
Pathology & $12(13.6 \%)$ \\
Glioblastoma & $2(2.3 \%)$ \\
Anaplastic astrocytoma & $1(1.1 \%)$ \\
Anaplastic oligoastrocytoma & $1(1.1 \%)$ \\
Anaplastic ependymoma & $1(1.1 \%)$ \\
Gliosarcoma & \\
Malignant glioma & $90(50,100)$ \\
KPS & \\
Median (min, max) & $64(72.7 \%)$ \\
Vital status & $26(27.3 \%)$ \\
Dead & \\
Alive & $402(37,2059)$ \\
Overall survival (days) & \\
Median (min, max) & $55(62.5 \%)$ \\
Insurance type & $33(37.5 \%)$ \\
Commercial & \\
Non-commercial* & \\
\hline
\end{tabular}

*Medicare (25), Medicaid (6), Healthy New York (2).

Overall, our patient cohort generated approximately 44,000 insurance claims from the date of diagnosis to the date of death for any reason, or the date the patient was last known to be alive, incurring total healthcare payments (median) of $\$ 184,159.83$ (95\%CI=\$151,214.98-\$222,431.36) (Table II). Among these payments, spending was highest for outpatient facilities $(\$ 64,628.88,95 \% \mathrm{CI}=\$ 56,108.61$ $\$ 78,285.72)$, followed by inpatient costs $(\$ 46,210.03$, $95 \% \mathrm{CI}=\$ 40,813.55-\$ 53,710.87)$, while costs for professional and prescription drugs were lower and nearly the same (\$22,069.09 and $\$ 22,119.39$, respectively) (Table II). When payments were further examined by service type, the highest costs (median) were found to be those of outpatient radiology $(\$ 46,294.91)$, followed by inpatient surgery $(\$ 30,786.47)$, prescription drugs $(\$ 22,102.29)$, inpatient medical care $(\$ 20,402.38)$ and pharmacy $(\$ 20,137.72)$ (Table II). As expected, the majority of outpatient radiology costs were for therapeutics (radiation oncology) $(\$ 40,938.71)$ while radiology costs for non-therapeutics (diagnosis) were $\$ 3,825.69$ (Table II).

Next, healthcare payments in each phase of care for these patients were studied. Unlike previous studies that used calendar months after diagnosis $(7,10,11,13,14)$, total costs were divided into three clinically relevant phases, i.e., 
Liu et al: High-grade Glioma Costs

Table II. Healthcare costs (median with 95\%CI) (\$) for HGGs by setting* and service type (number of patients).

\begin{tabular}{|c|c|c|c|c|c|c|c|}
\hline & Median & Lower & Upper & & Median & Lower & Upper \\
\hline Inpatient & $46,210.03$ & $40,813.55$ & $53,710.87$ & Professional & $22,069.09$ & $18,269.47$ & $24,734.59$ \\
\hline Surgical (70) & $30,786.47$ & $28,487.20$ & $33,686.99$ & Radiology (88) & $6,975.37$ & $5,959.88$ & $8,056.14$ \\
\hline Medical (71) & $20,402.38$ & $15,495.69$ & $26,558.72$ & Diagnosis (87) & $4,001.36$ & $3,000.51$ & $4,219.61$ \\
\hline SNF (13) & $8,100.00$ & $2,750.00$ & $14,143.08$ & Therapeutics (81) & $3,034.39$ & $2,476.05$ & $3,415.79$ \\
\hline \multirow[t]{2}{*}{ Maternity - Mother (1) } & $6,058.19$ & NA & NA & Inpatient Surgery (85) & $4,108.15$ & $3,434.57$ & $6,229.60$ \\
\hline & & & & Office/Home Visits (88) & $3,586.57$ & $2,925.25$ & $4,085.58$ \\
\hline Outpatient & $64,628.88$ & $56,108.61$ & $78,285.72$ & Allergy Immunotherapy (1) & $2,397.68$ & NA & NA \\
\hline Radiology (88) & $46,294.91$ & $40,725.44$ & $52,195.06$ & Inpatient Anesthesia (85) & $1,797.12$ & $1,530.00$ & $1,979.60$ \\
\hline Therapeutics (85) & $40,938.71$ & $35,756.13$ & $48,566.49$ & Hospital Visits (78) & $1,188.79$ & 977.17 & $2,219.88$ \\
\hline Diagnosis (79) & $3,825.69$ & $2,275.18$ & $5,139.95$ & Maternity - Non-Del. Services (1) & 934.88 & NA & NA \\
\hline Pharmacy (76) & $20,137.72$ & $7,189.46$ & $38,914.38$ & Maternity - Cesarean Deliveries (1) & 772.68 & NA & NA \\
\hline Non-chemotherapy (76) & $19,091.57$ & $7,033.47$ & $37,618.78$ & Outpatient Surgery (44) & 748.07 & 480.60 & $1,023.22$ \\
\hline Chemotherapy (52) & $2,605.52$ & $1,899.91$ & $3,477.01$ & Emergency Room Visits (81) & 583.23 & 422.79 & 672.40 \\
\hline Outpatient observation (8) & $2,362.66$ & 623.76 & $5,419.39$ & Physical Therapy (19) & 504.00 & 251.29 & $1,120.34$ \\
\hline Outpatient alcohol/drug (1) & $1,982.77$ & NA & NA & Outpatient Psychiatric (17) & 474.20 & 261.47 & $1,155.62$ \\
\hline Outpatient surgery (34) & $1,757.34$ & $1,258.59$ & $2,737.98$ & Allergy Testing (2) & 408.12 & NA & NA \\
\hline Emergency room (58) & $1,732.95$ & $1,303.82$ & $3,153.16$ & Outpatient Anesthesia (21) & 390.00 & 271.44 & 494.90 \\
\hline Pathology (86) & $1,567.36$ & $1,070.33$ & $2,001.14$ & Miscellaneous Medical (77) & 358.67 & 241.22 & 396.73 \\
\hline Other outpatient facility (85) & 980.09 & 762.37 & $1,197.97$ & Office Surgery (59) & 338.56 & 212.65 & 657.60 \\
\hline $\mathrm{PT} / \mathrm{OT} / \mathrm{ST}(30)$ & 934.49 & 530.61 & $1,362.79$ & Maternity - Anesthesia (1) & 328.80 & NA & NA \\
\hline \multirow[t]{2}{*}{ Cardiovascular (41) } & 79.47 & 72.49 & 85.96 & Chiropractor (17) & 295.69 & 174.23 & 987.00 \\
\hline & & & & Vision Exams (58) & 265.36 & 147.29 & 360.62 \\
\hline Prescription drugs & $22,119.39$ & $18,494.81$ & $27,059.70$ & Physical Exams (49) & 249.16 & 141.86 & 350.12 \\
\hline \multirow[t]{2}{*}{ Prescription drugs (83) } & $22,102.29$ & $18,095.19$ & $27,755.19$ & Pathology (67) & 155.40 & 104.22 & 321.56 \\
\hline & & & & Urgent Care Visits (27) & 140.00 & 119.63 & 190.12 \\
\hline Other & $5,364.84$ & $3,900.84$ & $8,200.91$ & Office Administered Drugs (77) & 136.12 & 89.75 & 182.98 \\
\hline Home health care (60) & $4,716.16$ & $3,164.89$ & $6,612.52$ & Immunizations (57) & 99.74 & 72.51 & 147.10 \\
\hline Ambulance (56) & $1,635.00$ & $1,090.40$ & $2,424.60$ & Hearing/Speech Exams (5) & 67.02 & 36.00 & 193.51 \\
\hline Unknown (9) & 434.00 & 153.36 & $1,106.00$ & Cardiovascular (81) & 56.93 & 38.22 & 113.39 \\
\hline Durable medical equipment (44) & 226.06 & 136.69 & 459.53 & & & & \\
\hline Glasses/Contacts (34) & 115.00 & 60.00 & 120.00 & & & & \\
\hline
\end{tabular}

Grand total

$184,159.83 \quad 151,214.98 \quad 222,431.36$

*Milliman Health Cost Guidelines. SNF: Skilled nursing facility; PT: physical therapy; OT: occupational therapy; ST: speech therapy; NA: not available.

initiation, maintenance and recurrence care. The leading cost was incurred in the initial treatment period $(\$ 66,673.80)$. After relatively low costs for maintenance care $(\$ 14,490.53)$, healthcare costs increased to $\$ 52,125.77$ for recurrence care (Figure 1). This is consistent with the intensity of treatment, i.e., intensive care is required for initiation and recurrence treatment for patients with HGGs.

In our patient cohort, most $(62.5 \%, 55 / 88)$ patients were under commercial insurance while the rest of the patients (37.5\%, 33/88) were under non-commercial insurance including Medicare, Medicaid and Healthy New York (Table I). Furthermore, patients with commercial insurance appeared to have longer survival time (411 days, $95 \% \mathrm{CI}=366.0-460.0$ days) compared with patients with noncommercial insurance (358 days, $95 \% \mathrm{CI}=220.0-658.4$ days) $(p=0.563)$ (Table III). The benefit of survival time for patients with commercial insurance was associated with higher number of medical claims (Table III), and higher healthcare costs $(\$ 235,732.85, \quad 95 \% \mathrm{CI}=\$ 218,933.12-$ $\$ 278,243.92)$ than those patients with non-commercial insurance $(\$ 142,134.07,95 \% \mathrm{CI}=\$ 98,623.82-\$ 148,634.70)$ $(p<0.001)$ (Table III). Additionally, higher healthcare costs in each setting and each phase of care were observed for patients under commercial insurance compared to those under non-commercial insurance including Medicare, Medicaid and Happy New York (Table III).

In order to investigate potential factors associated with healthcare costs for these patients, a GLM was run using total healthcare costs as dependent variable and age, gender, KPS, married status, insurance type and survival as predictors. Patients with commercial insurance and longer survival had significantly higher healthcare costs, and better KPS score was marginally correlated with higher healthcare costs. Age, gender and married status were not significantly 
associated with healthcare costs (Table IV). To further examine the robustness of our model, we ran sensitivity analyses including tumor grade and vital status, respectively. In both cases, similar findings were obtained, i.e., survival and commercial insurance were significantly associated with healthcare costs (data not shown).

\section{Discussion}

Despite the relatively small number of patients with HGGs, these patients have shorter survival time and incur higher healthcare costs compared to other types of cancers $(6,9)$. However, the majority of these studies were performed a decade ago. Two recent cost studies on HGGs, although with large number of patients from two commercially insured populations, didn't have detailed stage information and they used proxy criteria to identify GBM patients (presumed GBM), and many of their patients might have had low-grade histology $(10,11)$. Additionally, these studies, like many previous reports, did not have patient survival and performance (KPS) information. All this information is of utmost importance clinically as it impacts treatment regimen, and likely affects costs. In the present study, we identified patients with HGGs diagnosed and treated at our institution between January 1, 2011 and February 28, 2017, and linked patient clinical information from EHR with corresponding health insurance claims and payment data from Excellus BCBS. In this manner, we were able to individually review diagnosis, treatment, and payment data for each patient rather than relying on national databases with their inherent limitations. Total healthcare costs for HGGs were calculated from diagnosis to the date of death or the date the patient was last known to be alive. The median total healthcare payments for these patients were $\$ 184,159.83$ (95\%CI=\$151,214.98$\$ 222,431.36)$. The largest component of healthcare costs was outpatient services $(\$ 64,628.88$ with $95 \% \mathrm{CI}=\$ 56,108.61$ $\$ 78,285.72)$, followed by inpatient services $(\$ 46,210.03$ with $95 \% \mathrm{CI}=\$ 40,813.55-\$ 53,710.87$ ) (Table II). Furthermore, the highest cost was for outpatient radiology therapeutics (\$40,938.71) (Table II), consistent with a recent study showing that radiation therapy is the strongest contributor to costs for HGGs patients (11).

Furthermore, the healthcare costs were studied in different phases of care, i.e., initiation, maintenance and recurrence care. This way may be more clinically relevant, unlike previous studies which used calendar months after diagnosis $(7,10,11,13,14)$. Consistent with the intensity of clinical care in different phases, initial care of these patients incurred $\$ 66,673.80$, leading the total healthcare costs (Figure 1). This trend is in line with recent reports that the majority of healthcare costs for one-year postindex surgery for patients with malignant gliomas were incurred in the 6 months postindex surgery although total healthcare costs were not

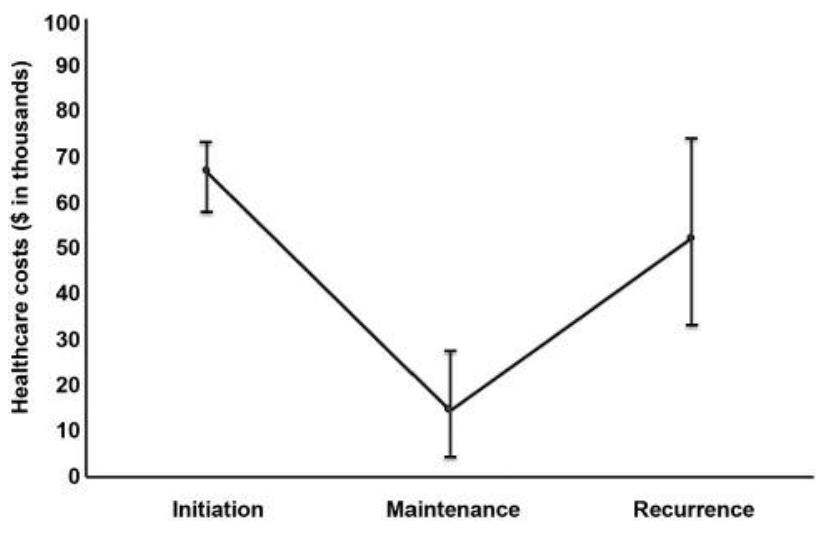

Figure 1. Healthcare costs by phase of care for the study cohort $(n=88)$. Data are shown as median $\pm 95 \%$ CI.

available in those studies $(10,11)$. Furthermore, the second peak of healthcare costs for these patients was during recurrence care $(\$ 52,125.77)$. Costs for maintenance care were relatively low $(\$ 14,490.53)$ (Figure 1). Such pattern has been reported in many types of cancers including brain cancer $(7,13,14)$.

It has been well documented that cancer patients with Medicaid or no insurance had a decreased likelihood of receiving advanced treatment, had greater burden of diseases and worse survival compared with those with private insurance (19-21). The impact of insurance status on malignant gliomas has been recently reported with mixed results. For example, Medicaid insurance and uninsured status were associated with shorter survival compared with nonMedicaid insurance for patients with $\operatorname{GBM}(22,23)$ and higher inpatient mortality after brain tumor resection (24). Similarly, Hooten et al. reported a higher patient safety indicators and hospital-acquired conditions for brain tumor patients with Medicare/self-pay compared with private insurance patients (25). In contrast, Kasl et al. observed that longer overall survival time for patients with GBM was associated with Veterans Affairs/Tricare/Medicaid insurance (26). In our study, approximately two-third of patients were under commercial insurance, this proportion is very close to state-wide and nation-wide brain tumor patients $(11,27)$. Furthermore, patients with commercial insurance appeared to have longer survival time compared to those with non-commercial insurance (411 days $v s .358$ days, $p=0.563$ ) (Table III), in line with a recent SEER study (22). In addition, patients with commercial insurance coverage had higher healthcare costs (\$235,732.85 vs. $\$ 142,134.07, p<0.0001)$ (Table III). This finding is supported by Chang's study that cancer patients (including brain cancer) with Medicare coverage had costs half that of commercial patients although no detailed clinical outcomes were available in their report (6). Similarly, Jiang et 
Table III. Comparisons between commercial and non-commercial segment cohort.

\begin{tabular}{lcc}
\hline & Commercial (55) & Non-commercial (33)* \\
\hline Gender & & $19(57.6 \%)$ \\
Male & $28(50.9 \%)$ & $14(42.4 \%)$ \\
Female & $27(49.1 \%)$ & $70(30,89)$ \\
Age (year) & $55(33,82)$ & \\
Median (min, max) & & $90(60,100)$ \\
KPS & $90(50,100)$ & \\
Median (min, max) & & \\
Healthcare costs (median with 95\%CI) (\$) & $54,675.52(46,157.01-72,413.63)$ & $29,727.76(21,416.41-42,188.36)$ \\
Inpatient & $87,841.00(66,439.04-114,240.96)$ & $46,079.81(24,674.15-51,398.57)$ \\
Outpatient & $27,121.10(24,144.93-33,310.11)$ & $13,420,47(10,975.67-15,761.69)$ \\
Professional & $25,710.30(22,102.29-30,633.15)$ & $11,793.07(8,185.12-19,524.73)$ \\
Prescription drugs & $7,562.82(4,122.69-10,807.53)$ & $3,748.76(1,661.87-7,965.82)$ \\
Other & $235,732.85(218,933.12-278,243.92)$ & $142,134.07(98,623.82-148,634.70)$ \\
Grand total & 514 & 460 \\
Number of claims per patient & & \\
Healthcare costs by phase of care (median with 95\%CI) (\$) & $81,159.92(72,505.13-89,294.47)$ & $53,426.03(39,751.45-61,898.82)$ \\
Initiation & $23,978.38(6,281.83-29,895.79)$ & $7,006.10(0.00-17,349.46)$ \\
Maintenance & $76,349.05(57,475.75-102,654.19)$ & $21,564.76(7,189.81-38,131.13)$ \\
Recurrence & $411.0(366.0-460.0)$ & $358.0(220.0-658.4)$ \\
Survival (median with 95\%CI) (days) &
\end{tabular}

*Non-commercial: Medicare (25), Medicaid (6), Healthy New York (2).

al. recently found that for GBM patients, commercial payer coverage was associated with more costs compared to noncommercial payer coverage (11).

In order to further identify drivers of healthcare costs for HGGs, a generalized linear model was run using healthcare costs as dependent variable and potential factors associated with healthcare costs as predictors. In line with a recent study (22), patients with commercial insurance had higher healthcare costs. In addition, patients with better KPS tended to have higher healthcare costs (marginally significant, Table IV). This is because patients with good KPS most likely receive aggressive treatments and thus, incur higher healthcare costs. More interestingly, it was also observed that patients with longer survival had higher healthcare costs. We further conducted two sensitivity analyses to examine the robustness of our model adding tumor grade and vital status, respectively, and obtained similar results. Higher healthcare costs didn't necessarily lead to better survival time for many types of cancer $(28,29)$ and correlation between healthcare costs and HGGs patient survival remains elusive. Our findings suggest HGGs patients at our institution received high-value care although relative low healthcare costs in our region compared to other areas of the nation (30).

Our study has several limitations. First, our study cohort was limited to our single institution and a single financial payer. However, our patient demographics and insurance coverage (Table I) is similar to regional and national data $(11,27)$, and thus our study may be representative of
Table IV. Results of GLM for healthcare costs for HGGs

\begin{tabular}{lccc}
\hline Variables & Beta & SE & Sig. \\
\hline Intercept & 11.773 & 0.5583 & 0.000 \\
Age & -0.007 & 0.0045 & 0.106 \\
Gender & & & \\
$\quad$ Male (Ref.) & -0.106 & 0.1008 & 0.294 \\
$\quad$ Female & & & \\
Married status & & & \\
$\quad$ Married (Ref.) & 0.033 & 0.1107 & 0.766 \\
$\quad$ Others & & & \\
Insurance type & & & \\
$\quad$ Commercial (Ref.) & -0.595 & 0.1154 & 0.000 \\
$\quad$ Non-commercial & 0.010 & 0.0052 & 0.057 \\
KPS & 0.001 & 0.0002 & 0.005 \\
Survival & & & \\
\hline
\end{tabular}

Ref.: Reference; Sig.: significance.

national trends. Second, healthcare costs in the present study were all paid by Excellus BCBS (based on the contractual rate). As such, our payments may be less than the total medical charge. Third, there is significant variation in healthcare costs in the US $(27,31,32)$ and healthcare costs in Rochester, NY are low compared to national benchmarks (30). Therefore, healthcare costs for HGGs in other regions of the nation are most likely higher than our data.

The strengths of our study include detailed clinical information i.e. individual review of diagnosis, treatment, 
performance status, and payment data for each patient, excluding those inherent limitations in national databases. Furthermore, the healthcare costs were divided based on clinical care for each individual patient, which we believe are more clinically relevant, unlike calendar months after diagnosis in the literature $(7,13,14)$. Additionally, rigorous methodology was used, not considered in previous studies $(6$, $7,9,10)$. The healthcare costs for these patients are substantial and high in the phases of initiation and recurrence care and relatively low between these two phases, suggesting that attempts to reduce costs are probably best focused on the period of disease recurrence where expenditures are high, but effectiveness seems low. Additionally, the significant administrative costs and patient burden of processing over 44,000 claims for 88 patients (500 claims/patient) represent an attractive target for improvement and a strong argument for payment bundling for disease management. Therefore, our findings are particularly useful for physicians, payers (public and private sectors) and policy makers to understand allocation of healthcare costs and provide high-value healthcare for these patients.

\section{Conflicts of Interest}

The Authors declare that they have no conflict of interest.

\section{Authors' Contributions}

YL: Study design, data collection and analyses, manuscript writing; ET: data collection and critical reading; ML: data collection and critical reading; DK: data collection and critical reading; KW: study design and manuscript writing.

\section{Acknowledgements}

The Authors would like to thank Yanju Julia Yu, Virak Nhek of Excellus BCBS for help in collecting claims data. They also thank Dr. Dongmei Li of University of Rochester for help with statistical analysis, Kwanza Warren of University of Rochester for help in collecting phase of care patient data, Linxi Liu of University of Rochester for help with data validation.

\section{References}

1 Ostrom QT, Gittleman H, Fulop J, Liu M, Blanda R, Kromer C, Wolinsky Y, Kruchko C and Barnholtz-Sloan JS: CBTRUS Statistical Report: Primary Brain and Central Nervous System Tumors Diagnosed in the United States in 2008-2012. Neuro Oncol 17: iv1-iv62, 2015. PMID: 26511214, DOI: 10.1093/ neuonc/nov189

2 Gittleman HR, Ostrom QT, Rouse CD, Dowling JA, de Blank PM, Kruchko CA, Elder JB, Rosenfeld SS, Selman WR, Sloan AE and Barnholtz-Sloan JS: Trends in central nervous system tumor incidence relative to other common cancers in adults, adolescents, and children in the United States, 2000 to 2010. Cancer 121: 102112, 2015. PMID: 25155924, DOI: 10.1002/ cncr.29015
3 Wen PY and Kesari S: Malignant gliomas in adults. N Engl J Med 359: 492-507, 2008. PMID: 18669428, DOI: 10.1056/ NEJMra0708126

4 Smith BD, Smith GL, Hurria A, Hortobagyi GN and Buchholz TA: Future of cancer incidence in the United States: burdens upon an aging, changing nation. J Clin Oncol 27: 2758-2765, 2009. PMID: 19403886, DOI: 10.1200/JCO.2008.20.8983

5 Stupp R, Mason WP, van den Bent MJ, Weller M, Fisher B, Taphoorn MJ, Belanger K, Brandes AA, Marosi C, Bogdahn U, Curschmann J, Janzer RC, Ludwin SK, Gorlia T, Allgeier A, Lacombe D, Cairncross JG, Eisenhauer E, Mirimanoff RO, European Organisation for R, Treatment of Cancer Brain T, Radiotherapy $\mathrm{G}$ and National Cancer Institute of Canada Clinical Trials G: Radiotherapy plus concomitant and adjuvant temozolomide for glioblastoma. N Engl J Med 352: 987-996, 2005. PMID: 15758009 , DOI: 10.1056/NEJMoa043330

6 Chang S, Long SR, Kutikova L, Bowman L, Finley D, Crown WH and Bennett CL: Estimating the cost of cancer: results on the basis of claims data analyses for cancer patients diagnosed with seven types of cancer during 1999 to 2000 . J Clin Oncol 22: 35243530, 2004. PMID: 15337801, DOI: 10.1200/ JCO.2004.10.170

7 de Oliveira C, Pataky R, Bremner KE, Rangrej J, Chan KK, Cheung WY, Hoch JS, Peacock S and Krahn MD: Phase-specific and lifetime costs of cancer care in Ontario, Canada. BMC Cancer 16: 809, 2016. PMID: 27756310, DOI: 10.1186/s12885016-2835-7

8 Blakely T, Atkinson J, Kvizhinadze G, Wilson N, Davies A and Clarke P: Patterns of cancer care costs in a country with detailed individual data. Med Care 53: 302-309, 2015. PMID: 25749656, DOI: 10.1097/MLR.0000000000000330

9 Kutikova L, Bowman L, Chang S, Long SR, Thornton DE and Crown WH: Utilization and cost of health care services associated with primary malignant brain tumors in the United States. J Neurooncol 81: 61-65, 2007. PMID: 16773215, DOI: 10.1007/s11060-006-9197-y

10 Ray S, Bonafede MM and Mohile NA: Treatment patterns, survival, and healthcare costs of patients with malignant gliomas in a large US commercially insured population. Am Health Drug Benefits 7: 140-149, 2014. PMID: 24991398

11 Jiang S, Hill K, Patel D, Waldeck AR, Botteman M, Aly A and Norden AD: Direct medical costs of treatment in newlydiagnosed high-grade glioma among commercially insured US patients. J Med Econ 20: 1237-1243, 2017. PMID: 28777020, DOI: $10.1080 / 13696998.2017 .1364258$

12 Tangka FK, Trogdon JG, Richardson LC, Howard D, Sabatino SA and Finkelstein EA: Cancer treatment cost in the United States: Has the burden shifted over time? Cancer 116: 34773484, 2010. PMID: 20564103, DOI: 10.1002/cncr.25150

13 Yabroff KR, Lund J, Kepka D and Mariotto A: Economic burden of cancer in the United States: Estimates, projections, and future research. Cancer Epidemiol Biomarkers Prev 20: 2006-2014, 2011. PMID: 21980008, DOI: 10.1158/1055-9965.EPI-11-0650

14 Mariotto AB, Yabroff KR, Shao Y, Feuer EJ and Brown ML: Projections of the cost of cancer care in the United States: 20102020. J Natl Cancer Inst 103: 117-128, 2011. PMID: 1228314, DOI: $10.1093 /$ jnci/djq495

15 American Society of Clinical Oncology: The State of Cancer Care in America, 2017: A Report by the American Society of Clinical Oncology. J Oncol Pract 13: e353-e394, 2017. PMID: 28326862, DOI: $10.1200 / J O P .2016 .020743$ 
16 Centers for Medicare \& Medicaid Services: Better Care. Smarter Spending. Healthier People: Paying Providers for Value, Not Volume, 2015.

17 Raizer JJ, Fitzner KA, Jacobs DI, Bennett CL, Liebling DB, Luu TH, Trifilio SM, Grimm SA, Fisher MJ, Haleem MS, Ray PS, McKoy JM, DeBoer R, Tulas KM, Deeb M and McKoy JM: Economics of Malignant Gliomas: A Critical Review. J Oncol Pract 11: e59-65, 2014. PMID: 25466707, DOI: 10.1200/ JOP.2012.000560

18 Burton E, Ugiliweneza B, Woo S, Skirboll S and Boaky M: A surveillance, epidemiology and end results-medicare data analysis of elderly patients with glioblastoma multiforme: treatment patterns, outcomes and cost. Mol Clin Oncol 3: 971978, 2015. PMID: 26623036, DOI: 10.3892/mco.2015.590

19 Pan HY, Walker GV, Grant SR, Allen PK, Jiang J, Guadagnolo BA, Smith BD, Koshy M, Rusthoven CG and Mahmood U: Insurance status and racial disparities in cancer-specific mortality in the United States: A population-based analysis. Cancer Epidemiol Biomarkers Prev 26: 869-875, 2017. PMID: 28183825, DOI: 10.1158/1055-9965.EPI-16-0976

20 Aizer AA, Falit B, Mendu ML, Chen MH, Choueiri TK, Hoffman KE, Hu JC, Martin NE, Trinh QD, Alexander BM and Nguyen PL: Cancer-specific outcomes among young adults without health insurance. J Clin Oncol 32: 2025-2030, 2014. PMID: 24888800, DOI: $10.1200 / \mathrm{JCO} .2013 .54 .2555$

21 Walker GV, Grant SR, Guadagnolo BA, Hoffman KE, Smith BD, Koshy M, Allen PK and Mahmood U: Disparities in stage at diagnosis, treatment, and survival in nonelderly adult patients with cancer according to insurance status. J Clin Oncol 32: 3118-3125, 2014. PMID: 25092774, DOI: 10.1200/JCO.2014.55. 6258

22 Rong X, Yang W, Garzon-Muvdi T, Caplan JM, Hui X, Lim M and Huang $\mathrm{J}$ : Influence of insurance status on survival of adults with glioblastoma multiforme: A population-based study. Cancer 122: 3157-3165, 2016. PMID: 27500668, DOI: 10.1002/ cncr.30160

23 Brown DA, Himes BT, Kerezoudis P, Chilinda-Salter YM, Grewal SS, Spear JA, Bydon M, Burns TC and Parney IF: Insurance correlates with improved access to care and outcome among glioblastoma patients. Neuro Oncol 20: 1374-1382, 2018. PMID: 29893906, DOI: 10.1093/neuonc/noy 102

24 Curry WT, Jr., Carter BS and Barker FG, 2nd: Racial, ethnic, and socioeconomic disparities in patient outcomes after craniotomy for tumor in adult patients in the United States, 1988-2004. Neurosurgery 66: 427-437, 2010. PMID: 20124933, DOI: $10.1227 / 01 . N E U .0000365265 .10141 .8 \mathrm{E}$
25 Hooten KG, Neal D, Lovaton Espadin RE, Gil JN, Azari H and Rahman M: Insurance status influences the rates of reportable quality metrics in brain tumor patients: a nationwide inpatient sample study. Neurosurgery 76: 239-247, 2015. PMID: 25603104, DOI: 10.1227/NEU.0000000000000594

26 Kasl RA, Brinson PR and Chambless LB: Socioeconomic status does not affect prognosis in patients with glioblastoma multiforme. Surg Neurol Int 7: S282-290, 2016. PMID: 27217966, DOI: $10.4103 / 2152-7806.181985$

27 Missios S and Bekelis K: Regional disparities in hospitalization charges for patients undergoing craniotomy for tumor resection in New York State: correlation with outcomes. J Neurooncol 128: 365-371, 2016. PMID: 27072560, DOI: 10.1007/s11060016-2122-0

28 Brooks GA, Li L, Sharma DB, Weeks JC, Hassett MJ, Yabroff $\mathrm{KR}$ and Schrag D: Regional variation in spending and survival for older adults with advanced cancer. J Natl Cancer Inst 105: 634-642, 2013. PMID: 23482657, DOI: 10.1093/jnci/djt025

29 Tannenbaum S, Soulos PR, Herrin J, Mougalian S, Long JB, Wang R, Ma X, Gross CP and Xu X: Regional medicare expenditures and survival among older women with localized breast cancer. Med Care 55: 1030-1038, 2017. PMID: 29068906, DOI: $10.1097 / M L R .0000000000000822$

30 Boissonnault $\mathrm{AB}$ : Where to find the nation's most affordable medical-care. 2015, May 31. http://www.myhealthfinder.com/ staticfiles/myHealthFinder-com_where_to_find_the_nations_ most_affordable_medical_care.Pdf.

31 Newhouse JP and Garber AM: Geographic variation in health care spending in the United States: insights from an Institute of Medicine report. JAMA 310: 1227-1228, 2013. PMID: 24008265, DOI: 10.1001/jama.2013.278139

32 Zygourakis CC, Liu CY, Yoon S, Moriates C, Boscardin C, Dudley RA, Lawton MT, Theodosopoulos P, Berger MS and Gonzales R: Analysis of cost variation in craniotomy for tumor using 2 national databases. Neurosurgery 81: 972-979, 2017. PMID: 28402457, DOI: 10.1093/neuros/nyx133
Received January 15, 2019

Revised January 23, 2019

Accepted January 24, 2019 